\title{
Implementing Trauma-Informed Care: Recommendations on the Process
}

\author{
Diane K. Yatchmenoff \\ Stephanie A. Sundborg \\ Mildred A. Davis
}

\begin{abstract}
The importance of trauma-informed care (TIC) is now recognized across most health and human service systems. Providers are calling for concrete examples of what TIC means in practice and how to create more trauma-informed organizations. However, much of the current understanding about implementation rests on principles and values rather than specific recommendations for action. This paper addresses this gap based on observations during the provision of technical assistance over the past decade in fields like mental health and addictions, juvenile justice, child welfare, healthcare, housing, and education. Focusing on the infrastructure for making change (the TIC workgroup), assessment and planning, and the early stages of implementation, the authors discuss barriers and challenges that are commonly encountered, strategies that have proven effective in addressing barriers, and specific action steps that can help sustain momentum for the longer term.
\end{abstract}

Keywords: Trauma-informed care; implementation; health and human services

Rates of past and current trauma are known to be high among service recipients involved in many health and human service systems (Hopper, Bassuk, \& Olivet, 2010; Ko et al., 2008; Salazar, Keller, Gowen, \& Courtney, 2013), in the social service workforce (Berger et al., 2012; Elliott, Bjelajac, Fallot, Markoff, \& Reed, 2005), and in the general population (Dube et al., 2005; Green et al., 2010; Huang, Schwandt, Ramchandani, George, \& Heilig, 2012; McLaughlin et al., 2010). Moreover, service providers recognize that not only are our public systems populated with trauma survivors but that many service settings, programs, and processes can be re-traumatizing (Bloom \& Farragher, 2011; Substance Abuse Mental Health Services Administration [SAMHSA], 2014a). Trauma-informed care (TIC) takes this understanding into account, based on the premise that when services feel safe, empowering, and welcoming for those affected by trauma, service recipients are more likely to engage in and benefit from care.

TIC is not an evidence-based intervention with fidelity measures and clearly outlined strategies, nor is there a single definition (Hopper et al., 2010). Experts agree, however, that essential components of TIC include awareness of the prevalence of trauma, understanding about the impact on service utilization and engagement, and commitment to incorporating those understandings in policy, procedure, and practice (Guarino, Soares, Konnath, Clervil, \& Bassuk, 2009; Hopper et al., 2010; SAMHSA, 2014a).

In recent years, efforts to define TIC, outline its principles, and generate buy-in have turned to a focus on implementation (Miller \& Najavits, 2012; Morrissey et al., 2014). Service providers are calling for concrete examples of what it means in practice and the most effective strategies at the organizational level to make the needed changes. However,

Diane K. Yatchmenoff, PhD, Former Director of Trauma Informed Oregon, Adjunct Faculty at the School of Social Work, Portland State University; Stephanie A. Sundborg, PhD, Research and Evaluation Coordinator for Trauma Informed Oregon, Portland State University; Mildred A. Davis, LCSW, PhD, Director of Trauma Informed Oregon, Associate Professor of Practice at the School of Social Work, Portland State University. 
despite a proliferation of national centers, conferences, proprietary models, web-based resources, training opportunities, and experts offering technical assistance or consultation, much of the dialogue about implementation remains academic, resting on principles and general guidelines (Baker, Brown, Wilcox, Overstreet, \& Arora, 2015).

In some cases, creating a more trauma-informed service system may be easy and intuitive. However, for many organizations, adoption of TIC is slow to take off and hard to sustain, despite deeply held interest and commitment (Hodgdon, Kinniburgh, Gabowitz, Blaustein, \& Spinazzola, 2013; Hopper et al., 2010). What is missing from the literature is detailed and concrete information about what commonly happens in the implementation process, the barriers that are encountered, factors that can facilitate the process, and how organizations are effectively moving forward despite significant challenges.

This paper addresses this gap, presenting observations from the provision of technical assistance over the past decade in fields like mental health and addictions, criminal justice, juvenile justice, child welfare, healthcare, housing, criminal justice, and education. The authors have consulted and/or facilitated a TIC change process for individual agencies, larger organizations, and inter-agency or cross-system initiatives. While these settings are all different and each organization within them is a unique entity, our experience suggests that too much has been made of these differences, while the reality is that there are significant common dimensions to the work, common obstacles that are encountered, and a common set of strategies that can facilitate progress.

The overarching process for the implementation of TIC (see, for example, Trauma Informed Oregon, 2016a) will be familiar to anyone who has used organizational planning tools in the past, such as the well-known Plan-Do-Study-Act (PDSA) model of iterative change (Institute for Healthcare Improvement, 2003). The PDSA cycle includes introducing the idea of change, forming a team, setting aims, establishing measures, selecting changes, testing and implementing changes, and then spreading changes. Similarly, for the trauma-informed care initiative in an organization, early steps include acquiring foundational knowledge, generating buy-in (Guarino et al., 2009; Hendricks, Conradi, \& Wilson, 2011; Institute for Health and Recovery, 2012; SAMHSA, 2014b), and ensuring other elements of readiness (Armenakis \& Harris, 2009) as a precursor to the assessment and planning phase. In this paper, we focus primarily on infrastructure (forming a team), assessment, and early steps in implementation, where difficulties most frequently arise and successes can mean the most.

Brief examples of action steps that organizations have taken to reflect the principles of trauma-informed care are mentioned in various sections below, but the overarching purpose of the paper is to provide support for the process of assessment, planning, and implementation rather than to detail specific trauma-informed practices that have been adopted.

\section{Infrastructure for Change: The TIC Workgroup}

Although we have seen some success with individual champions or small grassroots efforts that create a ripple effect in organizations, most successful TIC implementation initiatives begin with foundational training and subsequently the establishment of a 
workgroup that is charged with leading the implementation effort. Ideally, staff at all levels and from all roles in an organization receive training in the core knowledge areas: the nature of trauma; the impact of trauma on brain development and functioning; how trauma shows up in service systems; how systems may inadvertently re-traumatize or activate a trauma response; secondary and vicarious trauma in the workforce; and the definition and principles of trauma-informed care, including care of the workforce as well as the population seeking or using services.

A TIC workgroup is usually established soon after initial training. It has not worked particularly well, in our experience, when organizations have put this responsibility into existing structures such as quality improvement or safety committees. A team that is created specifically for this purpose has been more effective. The job of the workgroup (Guarino et al., 2009; SAMHSA, 2014b) includes:

- gathering information to identify strengths and challenges;

- recommending priorities for change;

- developing solutions or action steps in priority areas;

- monitoring results; and

- proposing additions or changes to agency policy to institutionalize traumainformed practices.

These primary purposes are generally well understood. What is sometimes overlooked is the role of the workgroup to sustain momentum across the organization and to model trauma-informed practice. For instance, if a workgroup forms and begins to meet but is not heard from again for six months, the opportunity to build on and enhance the initial impact of training is missed and staff buy-in may be lost. Moreover, even if the workgroup comes up with recommendations for changes and is successful in getting them instituted by management, the opportunity to demonstrate transparency and inclusiveness is missed if other staff feel unheard.

The most successful workgroups send out regular updates about membership, process, activities, priorities, and proposed recommendations, along with information about trauma or TIC (articles, video clips, fact sheets, etc.). These missives are also an opportunity to recognize exemplary practices observed in the workplace and to disseminate new ideas or concrete examples that staff can use. Some of our favorite workgroup communication tools have been electronic newsletters, but a simple email will suffice so long as it is regular, informative, and invites feedback. To encourage staff input, it can be helpful to create an email address for the workgroup. This serves the dual purpose of institutionalizing the group and ensuring that no one person is solely identified with the effort.

\section{Forming the Workgroup}

Workgroups can be challenging to manage and sustain. Some of the difficulties are preventable by considering the following as the initial team comes together.

Membership. It is axiomatic that workgroups represent different roles and different levels of authority in the organization (Guarino et al., 2009), but issues of relationship and power inevitably come into play. In large complex organizations, individuals will not 
necessarily know one another. One technician in a residential setting gently pointed this out when his expert outside consultant was confused by the awkward silences in the room. It helps to know ahead of time that the work of the first few months may progress slowly until relationships form.

More challenging are inevitable power differences if management or senior management is represented along with line staff, facilities staff, front office staff, etc. The advantages to including senior managers are manifold. Their direct involvement sends a message that TIC is valued at the highest levels. Moreover, the authority to make changes happen is also present; without that, workgroups can spin their wheels coming up with ideas and priorities that are subsequently ignored or rejected by the leadership or governance body. In some instances, we have seen strong resistance to including senior management because of significant trust issues. However, the workgroup is the place where some of these divisions can begin to be breached; sustaining factions only contributes to the problem and undercuts the effort.

It is helpful if workgroups include individuals with lived experience of trauma and of the organization or service system. In our experience, this rarely happens. More successfully, we have seen peer support personnel added to workgroups and consumer advisory groups involved in helping with the assessment and planning process (e.g., walking through the lobby or waiting areas; reviewing signage; providing perspective on what feels safe, welcoming, or otherwise in the agency's practices).

Considering the discussion and tasks involved, the optimal group is comprised of eight to ten members. However, workgroups may need to be larger to represent constituencies in the organization and to ensure reasonable attendance at meetings. If the group is larger, and attendance remains high, sub-committees can form for different tasks.

Recruitment and length of service. We have seen three approaches to recruitment: (a) the open invitation (anyone is welcome to join) which honors interest but may not achieve representation across the organization and may also result in an overly homogenous group with respect to views; (b) appointment by a senior manager or supervisors in different programs, which gains representation but could be perceived as favoritism and may not be representative of different perspectives; and (c) a slot-based application process with openings for representatives from different roles and levels, asking interested staff why they want to participate and what they bring to the process. In whatever way recruitment occurs, it is important to think through, ahead of time, the consequences and to have a process that is as transparent as possible. It has also been helpful to form the workgroup with a limited duration (six-eight months works well), at which point the group can revisit the structure, membership, and process. The TIC initiative will be ongoing (we have worked with organizations over four-five years in some cases) but membership in the workgroup need not be a long-term commitment. In fact, some organizations rotate membership regularly to give more staff a chance to participate, bring new ideas, and reduce the burden for individuals. It is important to have some continuity in the group, however, and a set of priorities to work from, so that it does not feel like starting over as the membership changes. 
Facilitation and technical assistance. Many workgroups find it helpful to have support, at least initially, from outside the organization. This can increase credibility in the early planning stages as well as help with structure and manage power differences or conflict. In small organizations or in those with knowledgeable staff, outside technical assistance may not be necessary. In the end, sustainability rests on internalizing the process. Ideally, an outside facilitator phases out as soon as possible, providing any additional consultation only periodically.

Even with outside assistance, someone in the organization will need to set up meetings, send out reminders, write agendas, facilitate the meetings, take minutes, etc. Management's commitment of staff time for a designated point person is important. This role can rotate, but we have found it works best when the point person is a committed champion for traumainformed care, is relatively well-versed in the concepts, has the ear of leadership, and is respected by colleagues.

\section{The Workgroup Process}

Forming the workgroup carefully may help avoid some of the common challenges to the process, but will not prevent others, especially those related to long-standing undercurrents in organizations with a history of trauma and oppressive practices. The condition of organizational trauma and its long-term impact has been described in detail (Bloom, 2010; Bloom \& Farragher, 2011). Briefly, this occurs when a system becomes fundamentally and unconsciously organized around the impact of chronic and toxic stress, such that the essential mission of the system is undermined. We find this phenomenon sometimes reflected in workgroup dynamics (Vivian \& Hormann, 2013). It is likely that any change process or introduction of innovation would run into the challenges related to organizational trauma, but the explicit focus on TIC (especially the open acknowledgment, often for the first time, of toxic stress and/or vicarious trauma in the workforce) almost certainly heightens its likelihood. This can show up in a variety of ways.

In systems like housing, child welfare, juvenile justice, community mental health, and others, there may be members of the workgroup who bring long-standing frustration with management or deeply held anger about perceived past wrongs; others may need to share their sense of being overwhelmed with the work itself or of not feeling seen, heard, and supported by managers or colleagues. For example, in a large complex public system, early successes with implementation were dismissed by one powerful workgroup member as not really addressing the important issues. This may have reflected a lack of shared vision and goals, but it also reflected deeper underlying issues. In one community mental health program, the TIC workgroup hung on through a very difficult phase (the facilitator said it felt like six months of group therapy) before they were ready to start work in earnest. If the workgroup cannot move past those feelings, however, the process breaks down. In some cases, it has been necessary to revisit group membership, goals, and expectations in order to restart processes that stalled out.

If there is a history of mistrust or finger pointing between different parts of the organization either across departments/programs or between line staff and management, this may show up as well. Silence in workgroup meetings can signal merely a lack of 
confidence or uncertainty about how to proceed but it may also reflect a lack of safety or strong resistance to working together. In some organizations, there can also be a sense of hopelessness or a lack of belief that anything will be different (a felt sense that initiatives come and go, are billed as promising but do not change anything).

All of this takes time to overcome and may sometimes be impossible (Bloom, 2012; Burnes, 2011), at least in the short run. In fact, there is probably a case to be made for holding off on a TIC initiative in some instances. However, we have seen TIC efforts in agencies struggle through ups and downs over a period of years, experience frustration and confusion in meetings, see changes in membership of the workgroup, have periods of inactivity, and still move forward. Organizations persist through these challenges when there is a commitment of key staff and leadership, understanding that it is a long haul process, solid relationships that exist or are built during the process, and - on a practical note-have strong facilitation skills available to the group. A set of guidelines for the workgroup (see, for example, Trauma Informed Oregon, 2016b) may help steer the process if the guidelines are reviewed, adapted as necessary, endorsed by the membership, and used regularly during meetings.

\section{The Planning Process}

The map of trauma-informed care is huge and without distinct boundaries. It can be confusing to know what is most important or even where to start. We have watched groups approach planning in a variety of ways over the years and based on some of their experiences, we recommend a hybrid approach that involves both responding to urgent/immediate felt concerns and simultaneously (or nearly simultaneously) using a more structured self-assessment process to provide an overall framework for the work. We discuss each of these approaches in turn.

\section{Dealing with the Immediate}

Based on our observations working with organizations over time, individuals join the TIC workgroup because there is something about trauma-informed care that resonates with them, either in their own experience personally (on or off the job) and/or in their experiences with the individuals they encounter in their work. It is important to honor, capture, and use this immediate sense of what is important. We often start the first workgroup by asking participants why they want to be in the workgroup and what they believe the organization can do to be more trauma-informed for both the workforce and individuals seeking or receiving services. Typically, we get enough material from this initial conversation to drive priorities for the first six months. However, it is also important to be sure that other staff have an opportunity to feed into the list of immediate issues, either during brainstorming sessions as part of training or in their teams, staff meetings, or focus/discussion groups convened for this purpose. In order for this to be useful, all staff need to have the basic language and ideas about trauma and trauma-informed care; foundational training is a prerequisite (Fallot \& Harris, 2009; Guarino et al., 2009).

In our experience, the issues that surface first are surprisingly similar across a wide range of organizations: 
- activated clients (in the waiting room, on the phone, in a confined office, or exam room) and the need for training in an effective and traumainformed response;

- lack of protocols for dealing with crisis situations (an incident of violence, a death, or police intervention) that in themselves can be extremely destabilizing to staff, individuals involved, bystanders; and

- the need for care of all concerned following such a crisis.

Other physical safety issues that affect staff (and sometimes clients) are also easy to elicit: simple things like poor lighting, or malfunctioning locks, or challenges accessing crisis services when the need arises. In complex organizations, issues also typically emerge related to communication and power dynamics within the system: lack of transparency in decision-making, personnel practices that generate anxiety, lack of communication and a sense of respect or mutuality between different programs or divisions within the organization, and lack of teamwork.

By acknowledging and working with the experience of the workforce immediately, the TIC initiative can gain buy-in. This is particularly true if there are simple steps that can be taken to resolve concerns that surface. Often these opportunities relate to physical safety. One primary healthcare clinic in an urban setting, for example, made immediate converts among the workforce by adding a combination lock and motion sensor light to the bike shed (located in a dark corner of the parking lot) and by creating a buddy system for individuals leaving the building at night to walk several blocks to their cars. In another case, a list of emergency numbers, printed and laminated, made staff feel safer. We call these high impact/low cost results. They are usually simple, inexpensive, and easy to accomplish, but they can make a big difference by sending a message that staff concerns are being heard.

The TIC worksheet. A worksheet of initial priorities can be helpful, especially if it includes a column for workforce hot spots and a column for circumstances that may activate those seeking or receiving services. If this document also notes how each issue relates to trauma (e.g., why it would be particularly activating for a trauma survivor, or what principle of trauma-informed care is involved), it can help keep the workgroup on topic. Often these two columns line up with the same activating circumstances affecting both populations. For example, an incident that happens in the lobby with a patient yelling or threatening the front office staff will have an impact on the staff but also on other patients who witness the event or hear about it later. Likewise, unexpected staff turnover or sudden changes in policy without warning may equally destabilize anxious workers and anxious patients/clients. This alignment can make it easier to advocate for a specific recommendation to senior management because it affects both populations and maintains a balance in priorities between the workforce and the people walking in the door for help. The worksheet is also a handy tool to come back to when discussion strays.

\section{Structured Assessment and Planning}

A systematic framework for assessment and planning provides a map of TIC that is recognizable and describable. By linking immediate concerns with longer-range goals, it 
is possible to connect an individual organization's efforts with those that are occurring across the system of care in the wider community. A structured framework makes it easy to communicate with stakeholders, including governance boards, staff, advisory groups, funders, etc., about what you are doing and where you are in the implementation effort. It can also help reassure even workgroup members that small steps are part of a bigger picture as well as providing an organized parking lot for the many ideas that are likely to surface.

Pioneer efforts by Harris and Fallot (2001), Jennings (2004), and others were followed by the development of formal guidelines and planning toolkits for organizations, frequently but not always focusing on particular service systems. They cover much the same territory and vary primarily with respect to their level of detail and the amount of emphasis on clinical practice versus a systems perspective. Fallot, Harris, and colleagues at Community Connections created one of the first and most comprehensive self-assessment and planning processes (Fallot \& Harris, 2006). The Center for Family Homelessness, likewise, created The Trauma-Informed Organizational Toolkit for Homeless Service (Guarino et al., 2009) that includes an assessment instrument. TIP 57 published by SAMHSA (2014b) is another comprehensive resource. Organizations all over the country presumably have been working with these or other tools for some time, with or without guidance from the authors or technical assistance from outside consultants. However, there is little in the literature about the experience or results from using these assessment tools.

Trade-offs with formal planning tools. In our early work with organizations, we often recommended either the planning tools developed by Community Connections or the Toolkit available from the National Center for Family Homelessness. Both are excellent; they can scarcely be improved on, with the exception of newer emphases that have emerged related to historical/cultural considerations, collective oppression/trauma, and the centrality of peer support.

We and our organizational partners have had mixed results. We found that the agencies that had turned to us for help or advice did not always have the capacity to take this route very effectively. First, the TIC effort is frequently led by a supervisor, a clinician who is passionately committed, or a behavioral health manager. Generally speaking, these individuals are working under tremendous pressure and, more important, come to the table with a sense of urgency and a helping perspective that can make the longer and more conceptual manuals or toolkits feel both daunting and not well-aligned with their immediate concerns. Given lack of confidence, lack of time, and limited support, the systems perspective was often not a good fit. Consequently, we had trouble getting workgroups to complete the longer manuals or toolkits.

The specific assessment instruments were more easily adopted since our partners recognized the need to gather information from staff and in some cases from volunteers and/or individuals receiving services. A number of organizations constructed surveys out of these tools, in some cases administering them to a large number of individuals. The process was lengthy and required substantial staff time in the development of the surveys to suit the context.

The bigger difficulty was in the output. These instruments yield huge amounts of information particularly if you tap into multiple sources, and it can be a long, arduous, and 
confusing process to sort through, report out, and use the data to guide priorities. Moreover, survey data usually resulted in numerical ratings. Average or summary scores can be hard to work with and give little help in determining what to do next. Finally, as agencies began to act on identified priorities and to make changes, they could report out on what they had accomplished, but they had no framework against which to gauge or report on their overall progress in a way that lined up with what other organizations might be doing.

Another challenge needs mentioning here as well. When an agency's service population are included in a large survey process, the intention is admirable but the data can be misleading. Consumer satisfaction surveys are known to have a strong positive response bias in most cases (Fowler, 2013; Patwardhan \& Spencer, 2012). More important, it is difficult to get reliable or valid data in a written survey from vulnerable individuals and especially if the population is diverse, potentially using English as a second language (or not at all), and without support and guidance around what is being asked and whether it is safe to answer honestly. It is a weak methodology at best.

As a result, we have taken to discouraging our partners from starting their assessment process with a survey unless it is highly targeted and based on at least a preliminary understanding about the current status. We also recommend that any survey should include open-ended questions inviting a qualitative response either in addition to or in instead of a set of ratings. If open-ended questions are not included or do not yield sufficiently useful information (it can sometimes be difficult to get participants to articulate their thoughts in text), following up with small group discussions to help interpret the results can be helpful.

At the other end of the spectrum are a number of checklists for TIC that can also be found online, and are often focused on general concepts ("our agency is committed to trauma-informed care") rather than concrete steps that have been taken. See, for example, National Council for Behavioral Health (2013) or U.S. Department of Health \& Human Services, Office of Adolescent Health (2015). These resources may be useful to stimulate thinking but they are necessarily crude and will rarely yield enough information to be useful in planning (or credible with stakeholders, since they are generally not wellanchored in specific or concrete detail and thus highly subjective).

The principles of TIC as framework. Another approach is to build the planning/assessment process around the principles of trauma-informed care. Again, despite differences in the language and the number of principles that are listed, there is strong congruence across the literature about the core principles. SAMHSA (2014a) now uses six principles; some of the original proponents outlined six or seven (see Hopper et al., 2010 for a review). New principles related to peer supports and to the critical importance of cultural responsiveness and sensitivity to gender issues have fleshed out areas that were underplayed in earlier work. Fallot and Harris (2009) modified their original highly detailed "Trauma-Informed Self-Assessment and Planning Protocol" (2006) to produce "Creating Cultures of Trauma-Informed Care (CCTIC): A Self-Assessment and Planning Protocol," organized around TIC principles, which we have seen used very effectively by small agencies with cohesive and like-minded staff.

In our work, it has been useful to collapse the principles of TIC into three major domains: safety, power, and self-worth. We base this framework on the understanding that 
traumatic experiences that have a lasting impact are those events that induce overwhelming fear, powerlessness, and, depending on the nature of the trauma, a sense of worthlessness. In light of this, the fundamental commitment of trauma-informed care is to avoid reinducing those experiences and instead to establish policies, practices, and procedures that, insofar as possible, create a safe context, restore power, and support self-worth. As reflected in Table 1, we find that larger sets of TIC principles fit readily into these areas, sometimes arguably into more than one.

\begin{tabular}{lll}
\multicolumn{2}{c}{ Table 1. Principles of Trauma-Informed Care: Agencies demonstrate Trauma-Informed Care } \\
& \multicolumn{2}{c}{ Through Practices, Policies, and Procedures } \\
\hline Restore Power Through: & Create Safe Context Through: & Build Self-Worth Through: \\
- Choice & - Physical safety & - Relationship \\
- Empowerment & - Trustworthiness & - Respect \\
- Strengths perspective & - Choice & - Compassion \\
- Skill building & - Transparency & - Mutuality \\
& - Predictability & - Collaboration \\
& - Clear and consistent boundaries & • Acceptance and nonjudgment \\
\hline
\end{tabular}

It is possible to use these domains for assessment as well. Physical safety has proven to be a good place to start. This domain is easily understood by workgroup members, and it is usually fairly concrete. Agencies are often doing a reasonably good job already regarding safety, so it is possible to highlight strengths as a starting point. Quite often, moreover, small fixes can make a big difference as noted earlier in the light on the bike shed example.

Moving beyond physical safety, however has been more difficult. Defining emotional safety can prove to be a stumbling block and is subject to highly individual interpretation. Issues of power and especially relationship, respect, mutuality, and so forth that constitute the self-worth domain can take a group into challenging areas, particularly in traumatized organizations with a particularly vulnerable workforce. Nonetheless, some agencies have worked successfully with the principles. Sample action steps based on this approach appear in Table 2.

For some organizations, a narrative approach (Clandinin \& Huber, 2010) can be appealing. In essence, workgroup members walk through the experience of a client from the moment the service need arises: the referral or self-referral, initial contact, appointment scheduling, entry and intake, the waiting room, location of bathrooms, signage, and so forth-all the way to exiting services. The idea is to look at each step along that path for conditions that might activate a trauma response, fail to activate a trauma response, or in fact may be welcoming/healing. This approach appeals especially to direct service staff because it is concrete rather than abstract and contains within it the experiences that clients may have shared with them. In contrast to more abstract assessment, the narrative approach lends itself easily and effectively to direct involvement from individuals with lived experience of trauma and of the service system in question. It can be useful, for example, for gathering information from consumer advisory groups or in listening sessions with service recipients. 
Table 2. Action Steps for Implementation Based on Key Principles of Trauma-Informed Care Create Safe Physical Context

- Crisis protocols in place \& practiced for critical incidents.

- Buddy system instituted for after-hours parking concerns.

- Lighting reviewed \& improved inside \& outside building.

- De-escalation training provided for all staff.

- Emergency numbers posted at every workstation.

Create Safe Emotional Context

- Intake forms reviewed $\&$ revised to be less activating/intrusive.

- Front office staff, security, custodial staff, \& direct service staff are taught how to recognize $\&$ respond to signs of trauma response in waiting room, office, lab, \& exam room. Scripts created \& practiced that are respectful \& de-stigmatizing.

- Client Handbook includes section about relationship between clinician \& client, explaining boundaries in a supportive \& informative way.

- Clients provided clear \& concrete information about what to expect at every juncture. A what you need to know document created \& available.

- Signage reviewed \& improved to be more welcoming \& clear.

- Restroom closed when mandatory tests (urinalysis) are underway.

- Gender inclusion signs posted.

- Staff debrief process created for critical incidents.

\section{Offer/Restore Power}

- Individuals provided whatever choices are available with respect to service options, scheduling, etc.

- Individuals asked at first appointment about their prior experience with the system \& their current needs.

- Staff \& clients regularly asked for feedback \& they also receive a report on what they said \& how it was addressed.

- Staff offered flexibility in work schedules.

- Peer support available \& offered.

Support Self-Worth

- Service recipients offered coffee or water (especially if staff are enjoying them).

- Observation \& appreciation of colleagues' trauma-informed work is routine.

- Self-care plans institutionalized as regular part of supervision.

- Staff can acknowledge strengths \& recognize the why behind behaviors, even if they are unacceptable; staff are able to connect the dots for clients about how trauma has impacted them.

- Diverse staff are hired to represent population served.

The middle way: Benchmarks. In recent years, new resources have been developed that fall somewhere between a crude checklist and the detailed set of highly individual issues that came out of the earlier assessment tools. "SAMHSA's Concept of Trauma and Guidance for a Trauma-Informed Approach" (SAMHSA, 2014a) falls into this category, reducing the content of "TIP 57" (SAMHSA, 2014b) to a 19-page document that includes questions or prompts for action that are organized into a set of ten implementation domains. Trauma Informed Oregon's "Standards of Practice for Trauma-Informed Care" (Trauma Informed Oregon, 2015) is another example of a benchmark approach, or the organizational self-assessment for youth residential programs developed at the University of South Florida (Hummer \& Dollard, 2010). Others combine elements in slightly different ways or in larger or smaller clusters, but the content is virtually identical. In each case, domains of concern typically include multiple organizational functions: governance, operations, human resources, physical environment, workforce development, service 
delivery, and program improvement/evaluation. When used appropriately, benchmarks can support a mezzo-level approach for management, guiding planning at a structural level while the workgroup continues to focus on specific micro-level concerns. Moreover, a set of specific standards or benchmarks can be used to highlight and communicate progress over time against a framework that is common across organizations. Sample benchmarks (Trauma Informed Oregon, 2015) are illustrated further in Table 3.

Table 3. Benchmarks for Trauma-Informed Care: Organized by Five Domains Common Across Systems Governance \& Leadership

- Board \& executive team attend training or information sessions to learn about trauma \& Trauma-Informed Care (TIC).

- Agencywide trauma policy is in place.

- Regular feedback from workforce \& service population is solicited \& used to improve practice.

- Resources are set aside to support mental \& physical health of staff \& to attend to vicarious or secondary trauma.

- Policy decisions are communicated with as much transparency as possible \& with a demonstrated understanding of the impact on staff.

\section{Physical Environment \& Safety}

- Physical environment has been reviewed \& modified to be as welcoming \& safe as possible (art work, signage, common areas, hallways, bathrooms); individuals with lived experience were part of that process.

- Crisis protocols in place; provision is made to ensure that no staff member is alone in the building.

- There is a designated space for staff to go when self-care or a time-out would be helpful.

\section{Workforce Development}

- Initial \& ongoing training about trauma \& trauma-informed care are institutionalized.

- Hiring practices (job descriptions, resume review, interview questions, etc.) reflect a commitment to traumainformed care.

- On boarding includes orientation to trauma-informed care \& the organization's commitment to it.

- Supervision \& performance reviews include expectation of ongoing learning \& application of TIC principles.

\section{Service Delivery}

- Required intake forms \& processes have been reviewed \& modified to reduce unnecessary detail or questions that could be activating for survivors.

- Easy-to-read materials are available that explain core services, key rules \& policies, \& procedure for questions, concerns, or complaints.

- [In direct service organizations], screening for trauma is routine \& trauma specific services are available.

- All staff understand heightened risk of suicide $\&$ are able to respond effectively or get appropriate help.

\section{Ongoing Systems Change}

- Infrastructure is in place to sustain ongoing assessment, planning, implementation, \& feedback.

- Staff time has been set aside to participate, including designated FTE for coordination.

- Clinic policies (new \& existing) are reviewed regularly for trauma-informed language \& content.

- Regular mechanism has been established to report out to entire organization about TIC practices \& the change process.

Benchmarks also may serve as a step towards accountability measures. More and more states are creating policies that require behavioral health or other entities to demonstrate that they are meeting minimum sufficient standards for TIC or are working towards them (see, for example, Oregon Health Authority, 2014). Standards or benchmarks are proving useful in demonstrating adherence to policy stipulations. In the long run, this work may also move us towards fidelity measures that would allow researchers to examine the impact of trauma-informed care (or "trauma-informed approach" as it is being rebranded) on the 
experiences and outcomes for the workforce and for individuals and families receiving services.

\section{Implementation and the Feedback Loop}

Setting priorities and agreeing on action steps may be the most time consuming and complex aspect of the work, but as organizations begin to implement specific changes, a number of new challenges frequently appear.

\section{Trauma-Informed Rollout}

Even the most well-conceived efforts to create a more trauma-informed organization can have unintended negative consequences if they are not carried out with skill and sensitivity. A new intake procedure, for example - one that slows the process down, eliminates unnecessarily triggering questions, provides more transparency and opportunity for clients to connect and engage - may be exemplary. However, it will feel anything but trauma-informed to staff if it is rolled out as a new policy that requires changes in the dayto-day work of already-stressed employees without warning, without providing enough time to adjust, or enough support for the potential impact on the workload.

Considering context. Along the same lines, if a trauma-informed policy fails to consider the context and realities of the workforce (or clients), it may result in a backlash. Schools are currently in the cross-hairs of the movement towards trauma awareness and improved practices. Many districts are seeking to change disciplinary policies and procedures to support students more humanely and effectively, recognizing that adverse experiences, past and present, are likely driving much student conduct. However, schools of education are not yet equipping teachers with the tools to de-escalate activated students or to manage their classrooms in new ways. If we ask teachers to respond differently, we need to have a strategy in place for when the child responds to "What happened to you?" rather than "What's wrong with you?" by throwing a chair across the room.

The needed skill set. Likewise, our human services field is famous for initiating promising new practices, putting them into policy, and failing to account for the skills and support needed to make these practices work as they are intended. Screening for adverse childhood experiences or a history of trauma is only one of many examples where some workers will be able to do it well initially, others may never have the skills, and some will need coaching and support over time. In our rush to implement TIC, we sometimes forget that there is a steep learning curve for many, even those that are enthusiastic about the ideas in the abstract.

Shared understanding of the concepts. Trauma-informed care relies on the operationalization of a set of principles rather than a manual with specific and well-defined action steps. This can lead to confusion. Concepts like transparency, for example, are easily misinterpreted. Does it mean telling a client everything you know or everything that has been said? This is a serious problem, and most organizations are not yet equipped to carry out the detailed discussions to ensure that everyone is on the same page about what is being asked of them. Some office staff in a juvenile detention facility in Wisconsin, for example, blamed trauma-informed care for an incident of violence and harm (Hall, 2016). Since 
physical safety is the first principle of TIC, on the face of it there was likely a misinterpretation (or over-interpretation) of one of the other principles.

Follow up. In small or very cohesive organizations, planning may lead directly and visibly to action, but in larger, more complex organizations, best laid plans do not always result in change. The workgroup may present a recommendation to management; there may even have been the opportunity for all staff to review or provide input about the draft protocol. The proposed solution may be put into policy and shared at an all-staff meeting. There is no guarantee, however, that it will be widely adopted across the organization.

One mental health organization initiated a large number of changes over a period of about three years based on recommendations from the TIC workgroup. The steps ranged widely and included creating an agency wide trauma policy, installing new and more welcoming signs in the lobby, involving clients in designing a planned remodel, requiring that staff self-care plans be included in supervision and reviewed annually, adding a section in the client handbook about boundaries so that clinicians could go over it with new clients together, initiating lunch and listen sessions where clients could bring ideas, concerns or grievances, and so on.

In a follow-up survey of staff, we learned that the required self-care reviews were only being used by about a third of the supervisors. Some of the newer staff had never heard of the policy. This is not because the organization failed in its efforts; it reflects only that culture change takes a long time, requiring vigilance and follow through. In other cases, laudable efforts may simply not be as visible to a busy and overworked staff. If there is a new crisis protocol that is brilliantly conceived, but staff do not know how to get to it easily when they need it, the impact will be minimal.

Impact. Assuming that a change actually occurred, there is no way to know without asking whether it makes a difference. At one of our partner agencies, for example, the leadership instituted a policy that every unit, department, team, or staff meeting across the entire multi-site system would start with appreciations, to set a positive tone and build community. In a follow up survey, we learned that although some teams seemed to do this well and some of the workforce told us it made a difference in their experience, a significant number of staff said it felt artificial, did not make a difference, or made things worse. This was important information for the workgroup to address.

Moving with resistance. No matter how solid the work, how well-intentioned, and how thoughtful the planning and implementation, there will be resistance to change (Choi, 2011; Oreg, Vakola, \& Armenakis, 2011). Not everyone gets it, not everyone will. Even when they do, it is challenging and stressful to be asked to do things differently. If organizations were to wait for $100 \%$ buy-in before they committed to an implementation process, nothing would happen. The most experienced advocates and consultants in the field (ACE Interface, 2014) recognize the importance of persistence - the capacity to move around resistance and continue to work with folks that are ready and available (Family Resources, 2013). We would add that it is necessary for leadership to support the process from the top - setting direction and even mandates. It is also necessary to include and involve every layer in the organization. But it still will not eliminate resistance-at least not entirely. You just keep going. 


\section{Down the Road}

As organizations begin to move further along this journey, we would like to see better documentation of the process and the results. Disseminating this information is useful in that it normalizes the challenges inherent in the work (and the time involved) and sparks ideas for other changes that can be made. We are also committed to developing evaluation resources and supports for organizations. The core questions are, first, What did we do and what happened? (the documentation piece); and then, If changes were implemented, what if any difference did it make to the experience of the workforce or those receiving services? For example, attention to trauma-informed care of the workforce might be reflected in reduced absenteeism, reduced turnover, greater sense of competence and confidence, and increased job satisfaction. Likewise, for those receiving services, trauma-informed policies, procedures, and practices could help improve, among other things, client/patient engagement and retention in services, rates of follow-through on appointments, buy-in with service plans, adherence to plan provisions, and reduced non-indicated use of emergency services.

These are merely examples of the kinds of outcomes that might appear on a logic model for evaluation of trauma-informed care. Research is stymied for the moment by the lack of a fidelity measure for trauma-informed care, a way to measure dosage (what changes, how many changes, what type of changes would influence these or other outcomes) and experience with the time element (how long it should take for outcomes to be realized). These are not simple questions and the answers are not going to come quickly. What is possible at this stage is documentation of our work-tracking the process, the outputs, and any difference we can detect both in terms of reported experiences of staff and clients and agency-level indicators of staff and client engagement. Careful documentation will pave the way for more rigorous research efforts aimed at demonstrating how the significant changes that are occurring across many service systems may be improving outcomes for children, adults, and families in our communities.

\section{References}

ACE Interface. (2014). Laura Porter. Retrieved from http://www.aceinterface.com/Laura_Porter.html

Armenakis, A. A., \& Harris, S. G. (2009). Reflections: Our journey in organizational change and research. Journal of Change Management, 9, 127-142. doi: https://doi.org/10.1080/14697010902879079

Baker, C. N., Brown, S. M., Wilcox, P. D., Overstreet, S., \& Arora, P. (2015). Development and psychometric evaluation of the Attitudes Related to TraumaInformed Care (ARTIC) scale. School Mental Health, 8(1), 61-76. doi: https://doi.org/10.1007/s12310-015-9161-0

Berger, W., Coutinho, E. S. F., Figueira, I., Marques-Portella, C., Luz, M. P., Neylan, T. C., . . .Mendlowicz, M. V. (2012). Rescuers at risk: A systematic review and metaregression analysis of the worldwide current prevalence and correlates of PTSD in rescue workers. Social Psychiatry and Psychiatric Epidemiology, 47(6), 1001-1011. 
doi: https://doi.org/10.1007/s00127-011-0408-2

Bloom, S. L. (2010). Organizational stress as a barrier to trauma-informed service delivery. In M. A. Becker \& B. L. Levin (Eds.), A public health perspective of women's mental health (pp. 295-311). Retrieved from http://www.sanctuaryweb.com/Publications/ListofPublications.aspx doi: https://doi.org/10.1007/978-1-4419-1526-9 15

Bloom, S. L. (2012). Trauma-organized systems. In C. R. Figley (Ed.), Encyclopedia of trauma (pp. 741-745). Retrieved from http://www.sanctuaryweb.com/Publications/ListofPublications.aspx doi: https://doi.org/10.4135/9781452218595.n255

Bloom, S. L., \& Farragher, B. (2011). Destroying sanctuary: The crisis in human service delivery systems. New York: Oxford University Press.

Burnes, B. (2011). Introduction: Why does change fail, and what can we do about it? Journal of Change Management, 11(4), 445-450. doi: https://doi.org/10.1080/14697017.2011.630507

Choi, M. (2011). Employees' attitudes toward organizational change: A literature review. Human Resource Management, 50(4), 479-500. doi: https://doi.org/10.1002/hrm.20434

Clandinin, D. J., \& Huber, J. (2010). Narrative inquiry. In B. McGaw, E. Baker, \& P. P. Peterson (Eds.), International encyclopedia of education (3rd ed., pp. 436-441). New York, NY: Elsevier. doi: https://doi.org/10.1016/B978-0-08-044894-7.01387-7

Dube, S. R., Anda, R. F., Whitfield, C. L., Brown, D. W., Felitti, V. J., Dong, M., \& Giles, W. H. (2005). Long-term consequences of childhood sexual abuse by gender of victim. American Journal of Preventive Medicine, 28(5), 430-438. doi: https://doi.org/10.1016/j.amepre.2005.01.015

Elliott, D. E., Bjelajac, P., Fallot, R. D. Markoff, L. S., \& Reed, B. G. (2005). Traumainformed or trauma-denied: Principles and implementation of trauma-informed services for women. Journal of Community Psychology, 33(4), 461-477. doi: https://doi.org/10.1002/jcop.20063

Fallot, R. D., \& Harris, M. (2006). Trauma-informed services: A self-assessment and planning protocol (Version 1.4). Washington, D.C: Community Connections. Retrieved from https://www.theannainstitute.org/TISA+PPROTOCOL.pdf

Fallot, R. D. \& Harris, M. (2009). Creating cultures of trauma-informed care (CCTIC): A self-assessment and planning protocol. Washington, DC: Community Connections. Retrieved from https://www.healthcare.uiowa.edu/icmh/documents/CCTICSelfAssessmentandPlanningProtocol0709.pdf

Family Resources [famresmarketing]. (2013, June 12). Laura Porter - dealing with resistance to trauma informed care [Video file]. Retrieved from https://www.youtube.com/watch?v=hupRddaE0tw 
Fowler, F. J., Jr. (2013). Survey research methods. Los Angeles, CA: Sage.

Green, J. G., McLaughlin, K. A., Berglund, P. A., Gruber, M. J., Sampson, N. A., Zaslavsky, A. M., \& Kessler, R. C. (2010). Childhood adversities and adult psychiatric disorders in the national comorbidity survey replication I: Associations with first onset of DSM-IV disorders. Archives of General Psychiatry, 67(2), 113123. doi: https://doi.org/10.1001/archgenpsychiatry.2009.186

Guarino, K., Soares, P., Konnath, K., Clervil, R., \& Bassuk, E. (2009). Trauma-informed organizational toolkit. Rockville, MD: Center for Mental Health Services, Substance Abuse and Mental Health Services Administration, the Daniels Fund, the National Child Traumatic Stress Network, and the W.K. Kellogg Foundation. Retrieved from http://www.air.org/resource/trauma-informed-organizational-toolkit

Hall, D. J., (2016, May). What role did trauma-informed care play in alleged juvenile prison abuse? WisconsinWatch.org. Retrieved from http://wisconsinwatch.org/2016/05/what-role-did-trauma-informed-care-play-inalleged-juvenile-prison-abuse/

Harris, M., \& Fallot, R. D. (Eds). (2001). Using trauma theory to design service systems. San Francisco, CA: Jossey-Bass.

Hendricks, A., Conradi, L., \& Wilson, C. (2011). Creating trauma-informed child welfare systems using a community assessment process. Child Welfare, 90(6), 187. Retrieved from http://www.chadwickcenter.org/CTISP/images/TICWPracticeToolkit.pdf

Hodgdon, H. B., Kinniburgh, K., Gabowitz, D., Blaustein, M. E., \& Spinazzola, J. (2013). Development and implementation of trauma-informed programming in youth residential treatment centers using the ARC framework. Journal of Family Violence, 28(7), 679-692. doi: https://doi.org/10.1007/s10896-013-9531-z

Hopper, E. K., Bassuk, E. L., \& Olivet, J. (2010). Shelter from the storm: Traumainformed care in homelessness services settings. The Open Health Services and Policy Journal, 3(2), 80-100. doi: https://doi.org/10.2174/1874924001003020080

Huang, M. C., Schwandt, M. L., Ramchandani, V. A., George, D. T., \& Heilig, M. (2012). Impact of multiple types of childhood trauma exposure on risk of psychiatric comorbidity among alcoholic inpatients. Alcoholism, Clinical and Experimental Research, 36(6), 1099-1107. doi: https://doi.org/10.1111/j.1530-0277.2011.01695.x

Hummer, V., \& Dollard, N. (2010). Creating trauma-informed care environments: An organizational self-assessment (in Creating trauma-informed care environments curriculum). Tampa, FL: University of South Florida, The Department of Child \& Family Studies within the College of Behavioral and Community Sciences. Retreived from

http://www.cfbhn.org/assets/TIC/youthresidentialself\%20assess\%20Fillable\%20FOR M\%20\%282\%29.pdf

Institute for Health and Recovery. (2012). Developing trauma-informed organizations: A tool kit. Retrieved from http://healthrecovery.org/images/products/30 inside.pdf 
Institute for Healthcare Improvement. (2003). The breakthrough series: IHI's collaborative model for achieving breakthrough improvement. Retrieved from http://www.ihi.org/resources/Pages/IHIWhitePapers/TheBreakthroughSeriesIHIsColl aborativeModelforAchievingBreakthroughImprovement.aspx

Jennings, A. (2004). Models for developing trauma-informed behavioral health systems and trauma-specific services. Alexandria, VA: National Association of State Mental Health Program Directors, National Technical Assistance Center for State Mental Health Planning. Retrieved from http://www.theannainstitute.org/MDT.pdf

Ko, S. J., Ford, J. D., Kassam-Adams, N., Berkowitz, S. J., Wilson, C., Wong, M., . . \& Layne, C. M. (2008). Creating trauma-informed systems: Child welfare, education, first responders, health care, juvenile justice. Professional Psychology: Research and Practice, 39(4), 396-404. doi: https://doi.org/10.1037/0735-7028.39.4.396

McLaughlin, K. A., Green, J. G., Gruber, M. J., Sampson, N. A., Zaslavsky, A. M., \& Kessler, R. C. (2010). Childhood adversities and adult psychiatric disorders in the national comorbidity survey replication II: Associations with persistence of DSM-IV disorders. Archives of General Psychiatry, 67(2), 124-132. doi: https://doi.org/10.1001/archgenpsychiatry.2009.187

Miller, N. A., \& Najavits, L. M. (2012). Creating trauma-informed correctional care: A balance of goals and environment. European Journal of Psychotraumatology, 3, 1-8. doi: https://doi.org/10.3402/ejpt.v3i0.17246

Morrissey, J. P., Jackson, E. W., Ellis, A. R., Amaro, H., Brown, V. B., \& Najavits, L. M. (2014). Twelve-month outcomes of trauma-informed interventions for women with co-occurring disorders. Psychiatric Services, 56, 1213-1222. doi: https://doi.org/10.1176/appi.ps.56.10.1213

National Council for Behavioral Health. (2013). Trauma-informed care-top ten: A checklist for behavioral health organizations. Retrieved from https://www.integration.samhsa.gov/clinical-practice/TIC_Flyer_web_2013_new.pdf

Oreg, S., Vakola, M., \& Armenakis, A. (2011). Change recipients' reactions to organizational change: A 60-year review of quantitative studies. The Journal of Applied Behavioral Science, 47(4), 461-524. doi: https://doi.org/10.1177/0021886310396550

Oregon Health Authority, Addictions and Mental Health Division. (2014). Trauma Informed Services (AMH-060-1607). Retrieved from http://www.oregon.gov/oha/HSD/AMH/Trauma\%20Policy/Trauma\%20Policy.pdf

Patwardhan, A., \& Spencer, C. H. (2012). Are patient surveys valuable as a serviceimprovement tool in health services? An overview. Journal Healthcare Leadership, 4, 33-46. doi: https://doi.org/10.2147/JHL.S23150

Salazar, A. M., Keller, T. E., Gowen, L. K., \& Courtney, M. E. (2013). Trauma exposure and PTSD among older adolescents in foster care. Social Psychiatry and Psychiatric Epidemiology, 48(4), 545-551. doi: https://doi.org/10.1007/s00127-012-0563-0 
Substance Abuse and Mental Health Services Administration [SAMHSA]. (2014a). SAMHSA's concept of trauma and guidance for a trauma-informed approach (HHS Publication No. SMA 14-4884). Rockville, MD: Substance Abuse and Mental Health Services Administration. Retrieved from http://store.samhsa.gov/shin/content/SMA14-4884/SMA14-4884.pdf

SAMHSA. (2014b). Trauma-informed care in behavioral health services. Treatment improvement protocol (TIP) series 57 (HHS Publication No. SMA 13-4801). Rockville, MD: Author. Retrieved from https://store.samhsa.gov/shin/content/SMA14-4816/SMA14-4816.pdf

Trauma Informed Oregon. (2015). Standards of practice for trauma informed care. Retrieved from http://traumainformedoregon.org/standards-practice-traumainformed-care/

Trauma Informed Oregon. (2016a). Roadmap to Trauma Informed Care. Retrieved from https://traumainformedoregon.org/roadmap-trauma-informed-care/

Trauma Informed Oregon. (2016b). Trauma Informed Care Workgroup Meeting Guidelines. Retrieved from https://traumainformedoregon.org/wpcontent/uploads/2016/01/Trauma-Informed-Care-Workgroup-MeetingGuidelines.pdf

U.S. Department of Health and Human Services, Office of Adolescent Health. (2015). A checklist for integrating a trauma-informed approach into teen pregnancy prevention programs. Retrieved from https://www.hhs.gov/ash/oah/sites/default/files/traumainformed-checklist.pdf

Vivian, P., \& Hormann, S. (2013). Organizational trauma and healing. North Charleston, SC: Create Space.

Address correspondence to: Diane Yatchmenoff, Portland State University, Regional Research Institute, 1600 SW Fourth Ave., Suite 900, Portland, OR 97201, yatchmd@pdx.edu 